\title{
Campus Virtual de la UNED: \\ Un Ejemplo de Aplicación de Nuevas Tecnologías en la Educación a Distancia
}

(Virtual Campus: New Technology Application Example in the Distance Learning)

\author{
Juan Peire \\ Manuel Castro \\ Africa López-Rey \\ Carlos De Mora \\ Clara Pérez \\ José Antonio Rodríguez \\ Universidad Nacional de Educación a Distancia
}

(España)

RESUMEN: La incursión de las nuevas tecnologías en entornos educativos, especialmente en aquellos de educación a distancia ha supuesto una auténtica revolución. El Campus Virtual es por definición el conjunto de funcionalidades que hacen posible la interacción entre los colectivos que componen la Universidad, sin necesidad de coincidir ni en el espacio ni en el tiempo. El proyecto Campus Virtual, continuación del proyecto DEMOS que fue iniciativa de la UNED, supone un entorno educacional donde la cooperación entre profesores, alumnos, tutores y expertos se realiza de modo flexible a través de la utilización de herramientas multimedia.

Entornos Distribuidos - Campus Virtual - Enseñanza a Distancia - Líneas RDSI

\begin{abstract}
The introduction of New Technologies in educational environments has promoted a real revolution. Virtual Campus involves a set of features that makes possible the interaction among different collectivities that belong to the University, but spatial and time independent. Virtual Campus project has been the continuance of a European Commision $4^{\text {th }}$. Framework Programme project called DEMOS, which was born as UNED initiative in 1996. Virtual Campus integrates an educational environment where cooperation among teachers, tutors, experts and student is carried out in a very flexible way through multimedia tools.
\end{abstract}

Distributed Environments - Virtual Campus - Learning Scenarios - ISDN Lines 


\section{INTRODUCCIÓN}

El fenómeno educativo es tan antiguo como la humanidad misma y no existe ni subsiste de una manera autónoma e independiente, sino más bien, todo lo contrario. La educación está estrechamente ligada al entorno y evoluciona con los acontecimientos de la sociedad en que se desarrolla. Es por ello, que todos los cambios y transformaciones que ha experimentado la sociedad actual han llevado a un replanteamiento y modernización de los sistemas educativos.

Entre los diversos cambios producidos destaca el rápido y profundo desarrollo tecnológico, el cual ha afectado a todos los aspectos de la vida, tanto individuales, como industriales, institucionales y gubernamentales. El empuje de las telecomunicaciones y los sistemas informáticos ha fomentado la investigación y la implementación de diferentes tipos de tecnologías (Brofferio, 1998). Sobre todo, estos avances han influido en el ámbito de la educación a distancia, puesto que abren nuevas posibilidades en la relación entre profesorado y alumnos.

\section{Entorno Virtual de Enseñanza-Aprendizaje}

Es un entorno adecuado para aplicar y reforzar técnicas del aprendizaje a distancia y aprendizaje cooperativo, usando de una forma eficiente todos los recursos tecnológicos disponibles. Las diferencias fundamentales entre estos nuevos entornos y los clásicos presenciales estriban en los canales de comunicación usados, en este caso, la telemática (Porter, 1997).

El Campus Virtual es por definición el conjunto de funcionalidades que hacen posible la interacción entre los colectivos que componen la Universidad (estudiantes, profesorado y personal de gestión) sin necesidad de coincidir ni en el espacio ni en el tiempo. El hecho de estudiar desde casa no implica estar desconectado de los servicios de la Universidad. De esta forma, los estudiantes pueden comunicarse con los estudiantes de otras Universidades, consultar los libros disponibles, entrar en el quiosco y consultar la prensa o poder preguntar a los profesores dudas sobre un tema. Este contacto es permanente entre los profesores y los estudiantes, lo que garantiza el estímulo constante que requieren las enseñanzas no presenciales.

El Campus Virtual se configura técnicamente como una Intranet asociada a una base de datos. Esta Intranet es accesible a través de distintos canales de comunicación (red telefónica básica, red digital de servicios integrados RDSI, InfoVía Plus, Internet, etc.) y facilita la intercomunicación entre todos los miembros de la comunidad universitaria (Hmelo, 1995).

Respecto a los beneficios de la tecnología en la educación, cabe destacar que la mayor parte de los estudios realizados señalan un efecto moderado y positivo en los logros de los estudiantes y una reducción substancial del tiempo de aprendizaje respecto a métodos tradicionales de enseñanza. Además aporta una serie de ventajas al proceso educativo (Castro, 1998), como pueden ser:

- Entrega multisensorial de la información: la enseñanza multimedia transmite la información a través de múltiples canales sensoriales, lo que permite a los estudiantes con distintos estilos de aprendizaje la asimilación y aplicación del conocimiento.

- Incremento de la propia expresión de los estudiantes y aprendizaje activo. Las nuevas tecnologías aportan entornos estimulantes que animan al estudiante a involucrarse en el proceso de aprendizaje.

- Razonamiento crítico. La tecnología puede promover una habilidad mayor de razonamiento de alto nivel. Algunos programas están diseñados específicamente para desarrollar las habilidades de resolución de problemas. El uso de hipermedia y telecomunicaciones también tiene su eco en las habilidades de razonamiento.

- Aprendizaje cooperativo. La introducción de la tecnología en el entorno de aprendizaje estimula una interacción mayor estudiante-profesor. 
- Habilidades de comunicación. El uso de tecnología en grupos pequeños incrementa las habilidades de comunicación. Permite también a estudiantes con discapacidades comunicarse con otros y expresarse por escrito.

- Educación multicultural. Las telecomunicaciones hacen posible expandir los límites del aula y comunicar a estudiantes y profesores tanto en el ámbito nacional como en el internacional. Estas interacciones capacitan a los estudiantes de distintos entornos culturales a construir puentes interculturales para estudiar problemas comunes desde distintas perspectivas. Aunque estos estudiantes pudieran comunicarse vía correo convencional, la realimentación a través de redes de ordenadores es normalmente más útil por su rapidez.

- Individualización y Personalización. Los estudiantes son diferentes y aprenden de distintas formas y a distinta velocidad. La tecnología ofrece diversidad a los estudiantes y un aprendizaje a su propio ritmo, permitiéndoles progresar a una velocidad adecuada y en un ambiente favorable.

- Motivación. Motivar a los estudiantes es un reto constante en educación. La tecnología puede inspirar a los estudiantes y profesores a realizar el aprendizaje de una forma excitante y relevante.

Pese a la anterior enumeración, cabe aclarar que la tecnología en sí no es el factor determinante en una educación efectiva. El punto importante es lo que se haga con la tecnología. Si ésta continua avanzando, tendremos que aprender a utilizarla sabiamente para motivar, enseñar, y retar a los estudiantes.

La Universidad Nacional de Educación a Distancia ha comprendido la necesidad de cambios y ha comenzado a introducir las nuevas tecnologías en su metodología de enseñanza. En el presente artículo se describe un proyecto cuyo objetivo principal era el desarrollo de aplicaciones enfocadas a la enseñanza a distancia sobre redes de telecomunicaciones.

\section{ESTRUCTURA DE LA UNED}

Según sus Estatutos, la UNED está constituida por Departamentos, Facultades y Escuelas Técnicas Superiores, Escuelas Universitarias y Centros Asociados. Los dos puntos clave de su estructura son la Sede Central y los Centros Asociados.

La Sede Central se encuentra en Madrid y es similar al resto de las universidades españolas, ya que consta de Facultades, Secciones y Departamentos.

Los Centros Asociados son las Instituciones básicas del Sistema y sirven de apoyo a las enseñanzas de la UNED. Son organismos autónomos que dependen de la Sede Central en lo que a los temas académicos respecta, si bien han sido creados por iniciativa local, y están patrocinados por corporaciones o entidades públicas o privadas. Todo alumno de la UNED ha de estar inscrito a un Centro Asociado, donde recibirá los servicios que se le ofrecen: información general, tutorías, convivencia, actividades culturales, prácticas de laboratorio, material didáctico, pruebas presenciales, biblioteca y mediateca, videoconferencia y televisión educativa (en algunos centros), matrícula y Órganos de Gobierno. Los Centros Asociados son, por tanto, el cauce a través del cual el alumno tiene relación con la Universidad y a su vez con sus compañeros.

El éxito docente de la UNED depende de la coordinación entre la Sede Central y los Centros Asociados. Así, la figura más representativa es la del profesor tutor, vínculo entre el Profesor responsable de la Sede Central y el alumno. El profesor tutor es el docente más característico de la enseñanza a distancia. Es una figura entre el profesor convencional y el de la Sede Central de una institución a distancia. A través de él se pretende personalizar la educación a distancia y ofrecer apoyo y asesoramiento al alumno. Sus funciones principales son la orientadora, tanto académica como personal y la de servir de punto de colaboración y nexo con la Sede Central. Para ello precisa una preparación específica en esa metodología. 
Por otro lado, la pluralidad metodológica se ha convertido en una de las características más peculiares de nuestro sistema (García Aretio, 1994). En este sentido nuestro objetivo es la integración del Campus Virtual como una herramienta más que se agregará a nuestro modelo educativo.

\section{MODELO DE CAMPUS VIRTUAL DE LA UNED}

Nuestro trabajo comenzó en enero de 1996 como socios del consorcio del proyecto DEMOS perteneciente al IV Programa Marco de I+D de la Unión Europea. El objetivo de DEMOS era el diseño y puesta a punto de un entorno educacional distribuido para escenarios de educación a distancia, donde la cooperación entre profesores, alumnos, tutores y expertos sea soportada de modo flexible mediante el uso de herramientas multimedia. Para ello se fijó como prioridad el desarrollo de aplicaciones educativas que fueran capaces de funcionar sobre entornos telemáticos muy heterogéneos.

El Centro de Servicios Informáticos (CSI) proporciona toda la infraestructura necesaria para posibilitar las comunicaciones a escala interna y externa (Carpio, 1994), de forma que se ponga en contacto la "Intranet" (red propia de la UNED, o RedUNED) existente en al UNED con la "Extranet" (red externa, o Red Corporativa) que abarca además a los Centros Asociados, y se engloba todo ello dentro de la red mundial, "Internet".

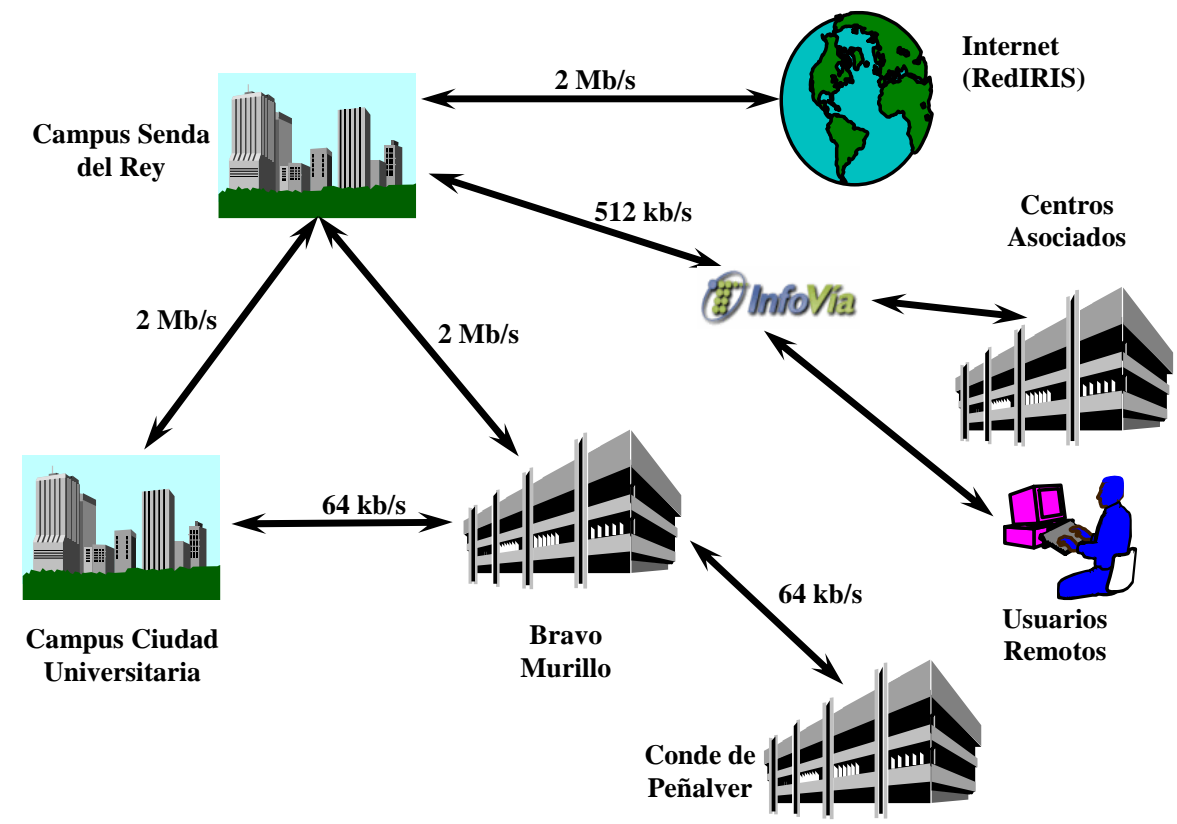

Figura 1. Red de comunicaciones en la UNED (principios de 1999)

La Figura 1 muestra el estado de las comunicaciones en la UNED a principios de 1999 y el estado previsto a mediados de dicho año se puede encontrar en la Figura 2, tras la realización del proyecto de integración de ATM en la RedUNED. 


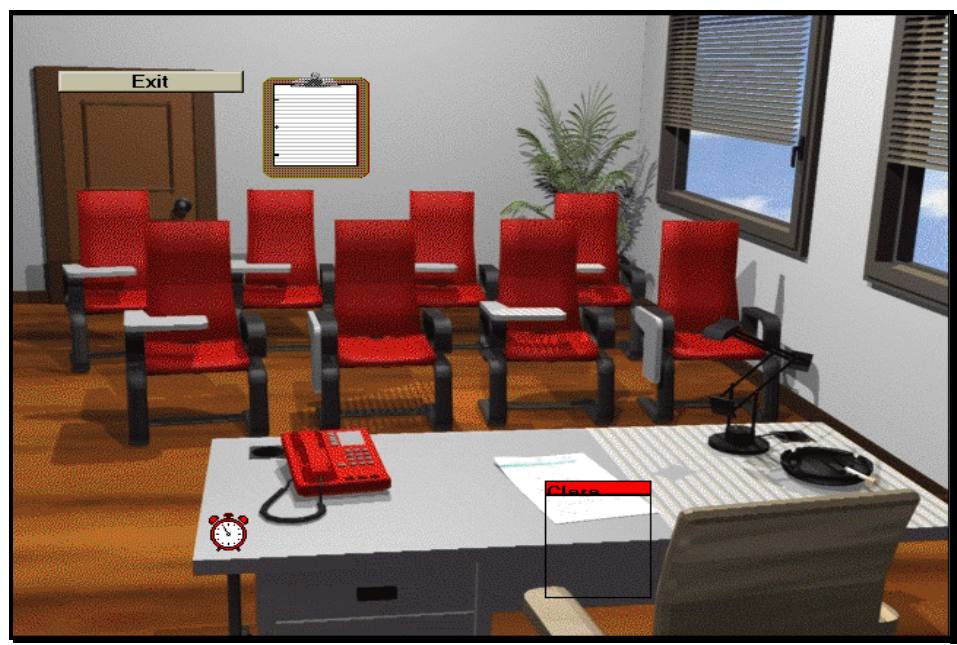

Figura 3. Clase Virtual Distribuida

- Por otro lado, el estudiante puede visitar el despacho virtual del profesor donde tendría lugar una TeleTutoría (Figura 4). Esta herramienta facilita la comunicación personal (punto a punto) entre el profesor y el estudiante: bien manteniendo una comunicación asíncrona, (como un correo electrónico pero con características multimedia), o bien estableciendo una comunicación en línea entre ambos (en este caso, podría entenderse como un uso punto a punto de la clase virtual).

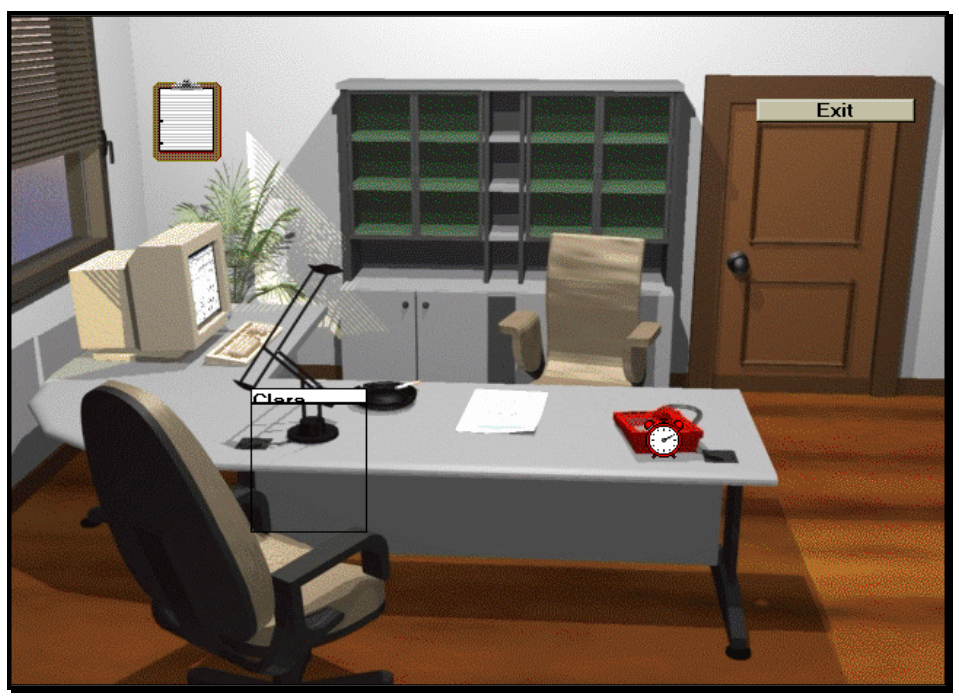

Figura 4. Despacho de Tele-Tutoría

- Finalmente, el estudiante puede visitar el Café-Room. Esta herramienta es un forum de discusión para los estudiantes, donde pueden mantener contactos vía correo electrónico y crear grupos de discusión, muy similares a los que existen en Internet. 


\subsection{DESCRIPCIÓN DE LOS DISTINTOS TIPOS DE USUARIOS}

El estudiante medio de la UNED es un hombre o una mujer que ronda los 30 años de edad, con un trabajo a tiempo completo y una familia, que quiere mejorar su perfil profesional de cara a lograr una promoción laboral o simplemente adquirir conocimientos para su desarrollo personal. Por supuesto, existe un importante número de alumnos jóvenes recién salidos de la enseñanza secundaria, y también muchos estudiantes maduros que están buscando obtener un segundo título universitario. Los estudiantes que trabajan cuentan con poco tiempo para dedicar al estudio y, por este motivo, tratan de aprovecharlo al máximo y sacarle todo el rendimiento posible. Por otro lado, los alumnos que viven en lugares remotos y aislados padecen un sentimiento de soledad que en muchos casos puede llevarlos al abandono de su tarea académica. A este tipo de estudiantes está especialmente dedicado este proyecto.

En cuanto al contexto de aplicación se distinguen principalmente dos tipos de usuarios potenciales:

a) Estudiante en casa. Este es un alumno típico de la UNED, vive cerca de un Centro Asociado pero las presiones laborales y familiares no le permiten acudir al Centro de forma frecuente. Desempeña su labor académica en casa, tiene un ordenador con características técnicas medias incluyendo algunos accesorios multimedia, entre los cuales podrían estar un módem de alta velocidad, tarjetas de vídeo y audio, o incluso de videoconferencia y posee acceso a la red del servicio telefónico standard (RTC), aunque considerando la evolución de los precios de los accesos a RDSI, este tipo de estudiante podría tener RDSI en su casa en un futuro no muy lejano.

b) Estudiante en un Centro Asociado. A pesar de que en la UNED no es obligatorio asistir a las tutorías, muchos estudiantes van de forma asidua a los Centros Asociados, especialmente aquellos que viven en ciudades de tamaño medio, pues para ellos no supone una inversión grande de tiempo ni dinero. Muchos de estos Centros Asociados ofrecen unas buenas instalaciones, incluyendo equipos informáticos bien dotados, acceso a RDSI y redes locales, y posibilidad de acceder a servidores UNIX de forma rápida y cómoda. Los estudiantes que cursan las mismas asignaturas tienen la oportunidad de encontrarse y trabajar juntos, y una vez a la semana cuentan con el soporte de un tutor durante todo el desarrollo del año académico.

\subsection{APLICACIONES DESARROLLADAS EN EL CAMPUS VIRTUAL}

En este apartado describiremos algunas de las aplicaciones que se han desarrollado en el proyecto Campus Virtual.

- Sistema de administración de los centros de enseñanza basado en el entorno WEB. Se ha definido una base de datos en la que se aparecen como entidades principales: los centros de enseñanza, los profesores y alumnos, los cursos que se imparten y la documentación asociado a cada curso. Además existen varios servicios de valor añadido entre los que cabe destacar la biblioteca y los tablones de anuncios. Para llevar a cabo esta aplicación se ha empleado la tecnología ASP (Active Server Pages) que permite confeccionar páginas HTML en tiempo real a partir de la información de diversas bases de datos. Para acceder a estas bases de datos, estamos utilizando RDO (Remote Data Objects) sobre ODBC.

- Compartición de documentos. Permite a los usuarios conectados a una sesión, visualizar y/o manipular, de forma sincronizada, documentos de diversa naturaleza, con objeto de colaborar en la realización de una determinada tarea. Se basa en la implementación de un núcleo central encargado de las comunicaciones y de varios visualizadores, uno por cada tipo de documento soportado. Actualmente han sido ya desarrollados visualizadores para textos (ASCII y RTF), imágenes (BMP, ICO, WMF, DIB, GIF, JPG), vídeos (AVI, MPG, MOV y, en general, todos aquellos formatos soportador por DirectMedia, audio (WAV, MDI), acceso remoto a información multimedia (RealAudio y RealPlayer, DirectShow), documentos de Office (PowerPoint, Word y Excel) y conversaciones CHAT. Para la implementación de estos componentes se utiliza la tecnología ActiveX, lo que permite utilizar como interfaz de usuario cualquier navegador de Internet que la 
soporte. Durante el presente año se ampliará, con objeto de mostrar la versatilidad del sistema, este conjunto de visualizadores en tres sentidos: aplicaciones matemáticas, simuladores y juegos.

- Compartición de aplicaciones. Permite a los usuarios ejecutar las mismas aplicaciones simultáneamente. Éstas se ejecutan en un solo terminal, pero son vistas y manipuladas desde varios. Existen dos posibilidades: en un primer caso, las aplicaciones que se ejecutan en un terminal son visualizadas en el resto de los terminales conectados (un profesor está explicando algún concepto a un grupo de alumnos), en un segundo caso, las aplicaciones que se están ejecutando en diversos terminales, son visualizadas en uno central (el profesor "ve" como trabaja un grupo de alumnos, de forma independiente, en una o varias tareas).

- Sistema de Tele-Presencia. Permite a los usuarios recibir y transmitir la señal de vídeo y/o audio captada por un cámara. Existen dos versiones de este componente. Por una parte un sistema de vídeo-conferencia estándar basado en la norma H.323. Este sistema, punto a punto, puede ser ampliado a una configuración multipunto utilizando un "VideoServer". Este tipo de instalación es bastante caro, pero asegura la interconexión entre los terminales del CAMPUS VIRTUAL y los del resto del mundo. Por otra parte existe un sistema de vídeo-conferencia multipunto sobre TCP/IP (basado en la transmisión de tramas de vídeo H.261 y H.263 y la transmisión de audio G.711, G.722 y G.728) de menor coste.

- Audio multipunto de alta calidad. Suministrado por AEQ permitirá a usuarios remotos transmitir con calidad $\mathrm{CD}$ una señal de audio de cualquier naturaleza. Actualmente $\mathrm{AEQ}$, dentro de otro proyecto ATYCA, está desarrollando un componente, denominado MiniCodec, capaz de realizar esta función. Este hardware tiene la ventana adicional de poder ser configurado, a través de líneas serie, de forma remota. Una de las tareas de este nuevo año, será la de desarrollar un componente software que realice esta configuración remota de manera automática según los parámetros suministrados por las aplicaciones de CAMPUS VIRTUAL.

- Servidor de comunicaciones. Aunque todas las aplicaciones comentadas anteriormente pueden funcionar sin la existencia de un servidor propiamente dicho (los mismos terminales clientes pueden actuar como servidores cuando esto es necesario), cuando nos referimos a redes de área extensa, como Internet, hay que tener en cuenta que la mayoría de los terminales están protegidos del exterior mediante Firewalls o sistemas Proxy. Este hecho impide la comunicación directa entre dos terminales conectados a Internet, lo que hace imprescindible la existencia de servidores que actúen como puentes entre los clientes. En este sentido, se ha desarrollado un servidor de comunicaciones que permite establecer conexiones multipunto entre un número no limitado de usuarios situados en localizaciones remotas. Para asegurar la versatilidad de este componente, se han realizado versiones para UNIX (Linux) y para Windows 95.

- Servicios de valor añadido. Se ofrece a los usuarios la posibilidad de grabar las sesiones de trabajo en grupo en ficheros. De esta manera, las lecciones podrán ser más tarde repasadas sin necesidad de establecer nuevas conexiones. Esta lección incluirá la documentación utilizada en la sesión, las acciones realizadas sobre ella por todos los usuarios del grupo y, opcionalmente, la señal de vídeo de dichos usuarios. Otro servicio a destacar es la gestión de listas de espera. Mediante ellas, es posible establecer horas de tutoría en las que los alumnos pueden consultar sus dudas los profesores. Por último, el sistema de vídeo-conferencia ha sido dotado con el servicio de contestador automático.

\subsection{ASPECTOS TÉCNICOS DEL CAMPUS VIRTUAL}

Desde un punto de vista técnico el Campus Virtual es un entorno telemático avanzado que incluye aplicaciones síncronas (on-line) y asíncronas (off-line). Puede ser ejecutado sobre diferentes plataformas hardware basadas en servidores PC multimedia, usando nodos punto a punto y multipunto.

El coste de la comunicación es uno de los criterios de aceptación más importante que afecta a los usuarios del Campus Virtual. La Red Telefónica Conmutada (RTC) puede ser empleada para conseguir un acceso por red de bajo coste (sobre todo para usuarios situados en áreas rurales), pero en la mayoría de los casos, la Red Digital de Servicios Integrados (RDSI) (Figura 5), que proporciona una velocidad de acceso de $128 \mathrm{kbps}$, es 


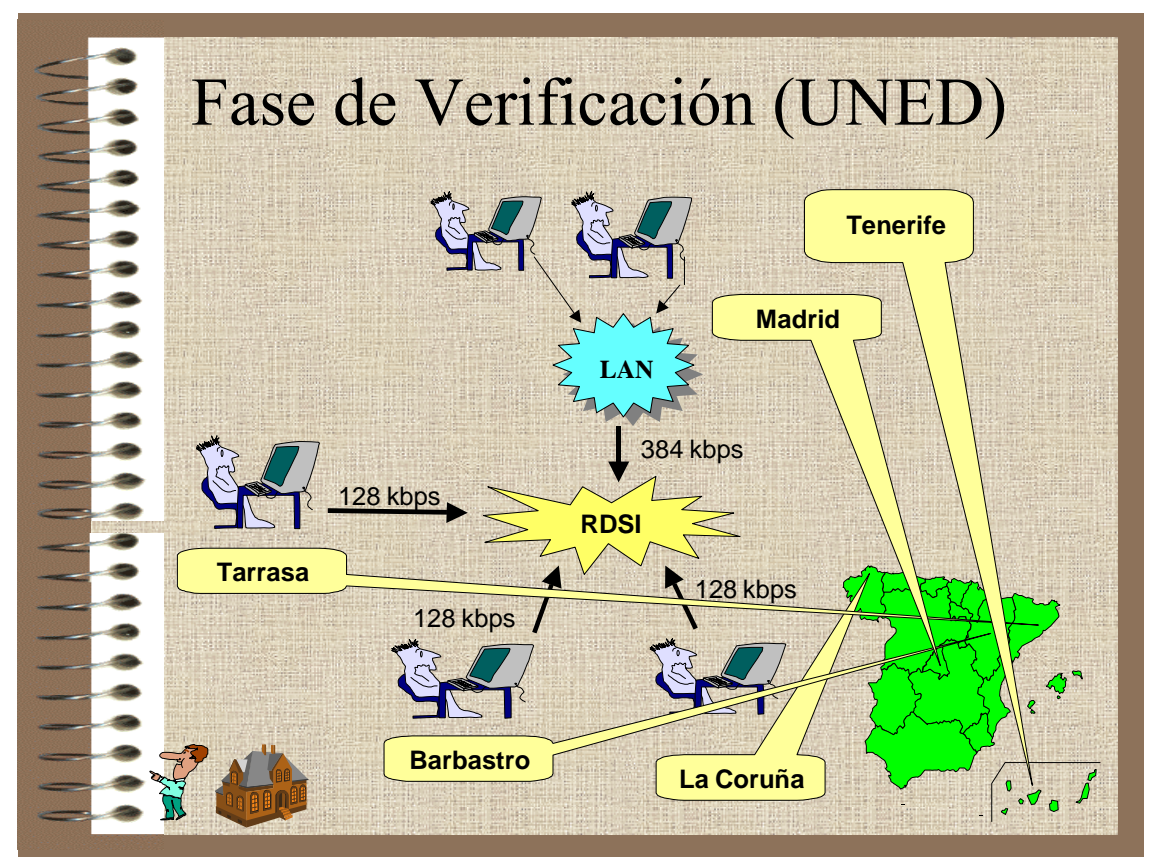

\subsection{VALIDACIÓN DEL PROYECTO}

El proyecto Campus Virtual ha prestado un interés especial a la elaboración del material didáctico que será empleado para impartir los cursos. Existe una aplicación destinada a la creación y puesta al día de los documentos multimedia que el profesor utilizará en sus explicaciones para los alumnos, principalmente en la Clase Virtual. Esta herramienta de composición ofrece un entorno amigable que permite la confección de los materiales para las clases de forma amena y sencilla, pudiendo incluir en los documentos toda clase de objetos y ficheros en distintos formatos (ficheros de texto, imágenes, sonido y vídeo), además, se da la posibilidad de trabajar con archivos que el profesor haya creado con otro tipo de programas basados en entorno Windows.

Después de considerar los tipos de usuarios, se ha pensado que dadas las características tan peculiares de la UNED, el Centro Asociado es el lugar más racional para la validación y demostración de un nuevo programa educativo. Esto es así porque aunque los estudiantes no asistan regularmente a las tutorías que tienen lugar en los Centros, o no saquen todo el partido que se podría de los servicios que allí se les ofrece, los estudiantes están ligados a los Centros al menos en el sentido administrativo, y además tienen la obligación de asistir para poder examinarse o realizar seminarios prácticos.

Las fases de demostración y validación se realizaron con estudiantes de los Centros Asociados de La Coruña, Barbastro, Tenerife y Tarrasa. Los esquemas de conexión pueden verse en la Figura 6.

Figura 6: Fase de Verificación del proyecto.

De las experiencias realizadas se llegó a la conclusión de que era preciso contar un técnico en cada punto de destino (en este caso en el Centro Asociado) que estuviera capacitado para realizar las conexiones necesarias en el equipo. Además, deben de estar perfectamente documentados los pasos que se han de seguir para la instalación de la aplicación en el equipo. 


\section{CONCLUSIONES}

El proyecto Campus Virtual supone una gran innovación para la educación a distancia, puesto que alivia la sensación de soledad de los estudiantes de zonas aisladas, mejorando su motivación y por lo tanto su rendimiento en el estudio. Los estudiantes pueden establecer una comunicación personal tanto con el profesor como con otros compañeros de forma semejante a la Universidad tradicional.

Debido a la gran diversificación de alumnos de la UNED, todas las aplicaciones ofrecidas por Campus Virtual se han adaptado a una infraestructura que permitirá establecer comunicaciones tanto punto a punto como multipunto a través de RDSI y RTC, incrementando el número de beneficiarios potenciales. Además de las ventajas que nos ofrece un entorno multimedia de trabajo, la innovación más importante del proyecto es la utilización de tarjetas de vídeo-conferencia para un programa de Enseñanza a Distancia Distribuida, con el consiguiente apoyo pedagógico que supone para los alumnos el hecho de poder disponer de la imagen del profesor que está impartiendo la clase, como si de una clase convencional se tratase. Se ha conseguido que las aplicaciones tuvieran una interfaz cómoda y sencilla que redujera al mínimo el periodo de aprendizaje de la herramienta y que ayudara a concentrarse en el proceso de aprendizaje en vez de en la manera de usar la aplicación.

\section{REFERENCIAS BIBLIOGRÁFICAS}

BrofFERIO, S.C. (1998) A University Distance Lesson System: Experiments, Services, and Future Developments. IEEE Transactions on Education, Vol. 41, No. 1, pp. 17-24.

CARPIO, J y RAmos, E. (1994). UNED-EVCN: UNED Educational Videoconferencing Network. DELTA Conference for Education and Training.

Castro, M. y otros. (1998). Distance Learning in the European Comunity. IEEE Transactions on Education (pendiente de publicación).

GARCiA ARETIO, L. (1994). La Educación a Distancia Hoy. Ed. UNED.

Hmelo, C.E. y otros. (1995). Multimedia Courseware for Teaching Dynamic Concepts: Assessment of Students Learning. Frontiers in Education $25^{\text {th }} \cdot$ Annual Conference. IEEE Catalog No. 95CH35867.

PÉrez, C. y DE MorA, C. (1997). Construcción de aulas virtuales como medio de soporte para la educación a distancia. Congreso Internacional de Informática Educativa. UNED

PORTER, L.R. (1997) Creating the Virtual Classroom. Chapter 2. USA. John Wiley Sons.

SÁNCHEZ, G. (1994). EDUBA: Applications for Education on Wide Band Communication Networks. DELTA Conference for Education and Training.

\section{PERFIL ACADÉMICO Y PROFESIONAL DE LOS AUTORES}

Juan Peire Arroba: Doctor Ingeniero Industrial por la Escuela Técnica Superior de Ingenieros Industriales de la Universidad Politécnica de Madrid y Licenciado en Derecho por la Universidad Complutense. Actualmente es Catedrático de Universidad del área de Tecnología Electrónica en el DIEEC, ETSII de la UNED, a la vez que es Director del Departamento. Ha trabajado varios años como Consultor especializado en la creación de Empresas Tecnológicas, así como ha dirigido y dirige diversos proyectos de investigación tanto nacionales como internacionales. Es miembro del IEEE.

Juan Peire Arroba

DIEEC/UNED

Ciudad Universitaria $\mathrm{s} / \mathrm{n}$

28040 MADRID

Tlf: +34913986480

Fax +34913986028

e-mail: jpeire@ieec.uned.es

Manuel Castro Gil: Doctor Ingeniero Industrial por la Escuela Técnica Superior de Ingenieros Industriales de la Universidad Politécnica de Madrid e Ingeniero Industrial, especialidad Electricidad, intensificación Electrónica y Automática por la misma Escuela. Actualmente es Profesor Titular del área de Tecnología Electrónica en el DIEEC, ETSII de la UNED, a la vez que es Director del Centro de Servicios 
Informáticos de la UNED. Ha trabajado cinco años en Digital Equipment Corporation. Es miembro Senior del IEEE y del consejo de dirección de ISES España.

e-mail: mcastro@ieec.uned.es

Africa López-Rey García-Rojas: Ingeniera Industrial, es becaria predoctoral en el Departamento de Ingeniería Eléctrica, Electrónica y de Control de la ETSII de la UNED participando en diferentes actividades docentes e investigadoras que desarrolla el Departamento.

e-mail: alopez@ieec.uned.es

Carlos de Mora Buendía: Doctor Ingeniero Industrial por la Universidad Politécnica de Madrid (UPM) y Profesor Titular del área de Ingeniería de Sistemas y Automática en el DIEEC E.T.S. de Ingenieros Industriales de la UNED. Está especializado en control de procesos con computador, incluyendo la utilización de técnicas de inteligencia artificial. Es experto en Sistemas de Gestión de Bases de Datos y ha impartido diversos cursos sobre control, bases de datos y programación de computadores.

e-mail: cdemora@ieec.uned.es

Clara Pérez Molina: Licenciada en Ciencias Físicas por la Universidad Complutense de Madrid en el año 1995. Actualmente está inscrita en el Programa de Tercer Ciclo del DIEEC de la UNED, donde trabaja como becaria. Ha participado en el desarrollo del proyecto DEMOS y es miembro del equipo del Campus Virtual. Sus intereses como investigadora abarcan los entornos telemáticos dedicados a la Educación a Distancia, los sistemas de manejo de información y las redes neuronales.

e-mail: clarapm@ieec.uned.es

José Antonio Rodríguez Criado: Ingeniero de Telecomunicaciones, es profesor ayudante en el Departamento de Ingeniería Eléctrica, Electrónica y de Control de la ETSII de la UNED participando en diferentes actividades docentes e investigadoras, tanto en enseñanza a distancia como en simulación y planificación de sistemas eléctricos basados en energías renovables.

e-mail: jarguez@ieec.uned.es 